\title{
Correction to: Optimization of Controllable Factors in the Aluminum Silicon Eutectic Paste and Rear Silicon Nitride Mono-Passivation Layer of PERC Solar Cells
}

\author{
Sungeun Park ${ }^{1} \cdot$ Hyomin Park ${ }^{1} \cdot$ Dongseop Kim² JungYup Yang $^{3} \cdot$ Dongho Lee $^{2} \cdot$ Young-Su Kim $^{2} \cdot$ Hyun-Jong Kim $^{2} \cdot$ \\ Dongchul Suh ${ }^{4} \cdot$ Byoung Koun Min ${ }^{5} \cdot$ Kyung Nam Kim ${ }^{6} \cdot$ Se Jin Park ${ }^{1}$. Donghwan Kim ${ }^{1} \cdot$ Hae-Seok Lee ${ }^{6}$. \\ Junggyu $\mathrm{Nam}^{2} \cdot$ Yoonmook Kang ${ }^{6}$
}

Published online: 28 June 2018

(c) The Korean Institute of Metals and Materials 2018

\section{Correction to: Metals and Materials International (2018) 24:664-671 https://doi.org/10.1007/s12540-018-0032-8}

The original version of this article unfortunately contained a mistake. The acknowledgements section was incomplete. The correct information is given below:
Acknowledgements This work was supported by a National Research Foundation of Korea Grant funded by the Korean Government (MSIP) (2016, University-Institute 165 cooperation program) and the New \& Renewable Energy Core Technology Program of the Korea Institute of Energy Technology Evaluation and Planning (KETEP), granted financial resource from the ministry of Trade, Industry \& Energy, Republic of Korea (No. 20163010012430) and the Energy Technology Development Program of the Korea Institute of Energy Technology Evaluation and Planning (KETEP) Grant (No. 20178520000470).

$\triangle$ Yoonmook Kang

ddang@korea.ac.kr

1 Department of Materials Science and Engineering, Korea University, Seoul 02841, Republic of Korea

2 Solar Energy Development Group, Samsung SDI, Yongin 17084, Republic of Korea

3 Department of Physics, Kunsan National University, Kunsan 54150, Republic of Korea

4 Department of Chemical Engineering, Hoseo University, Asan 31499, Republic of Korea

5 Clean Energy Research Center, Korea Institute of Science and Technology, Seoul 02792, Republic of Korea

6 KU-KIST Green School, Graduate School of Energy and Environment, Korea University, Seoul 02841, Republic of Korea 